\title{
Efeitos do treinamento de força na flexibilidade de mulheres idosas
}

\author{
Artigo Original
}

\section{Rodrigo Gomes de Souza Vale}

PROCIMH - Universidade Castelo Branco / RJ - Brasil

LABIMH - UCB / RJ

Grupo de Desenvolvimento Latino-Americano para a Maturidade - GDLAM vale@redelagos.com.br

Juliana Boscher Torres

\section{PROCIMH - Universidade Castelo Branco / RJ - Brasil}

LABIMH - UCB / RJ

Docente da Universidade Estacio de Sá - UNESA

torres-juliana@ig.com.br

\section{Karina Oliveira Martinho}

PROCIMH - Universidade Castelo Branco / RJ - Brasil

LABIMH - UCB / RJ

karinamartinho@tdnet.com.br
Rafaella Bauerfeldt Lopes

PROCIMH - Universidade Castelo Branco / RJ - Brasil

LABIMH - UCB / RJ

rblvasco@terra.com.br

Jefferson da Silva Novaes

PROCIMH - Universidade Castelo Branco / RJ - Brasil

Laboratório de Biociências da Motricidade Humana LABIMH - UCB / RJ

Universidade Federal do Rio de Janeiro (EEFD-RJ) - Brasil;

Bolsista da FUNADESP - SP / Brasil

jnovaes@terra.com.br

\section{Estélio Henrique Martin Dantas}

PROCIMH - Universidade Castelo Branco / RJ - Brasil

LABIMH - UCB / RJ

Grupo de Desenvolvimento Latino-Americano para a Maturidade - GDLAM

Bolsista de Produtividade em Pesquisa - CNPq

estelio@cobrase.com.br

VALE, R.G.S.; TORRES, J.B.; MARTINHO, K.O.; LOPES, R.B.; NOVAES, J.S.; DANTAS, E.H.M. Efeitos do treinamento de força na flexibilidade de mulheres idosas. Fitness \& Performance Journal, v.3, n.5, p. 266-271, 2004.

Resumo: $\bigcirc$ objetivo deste estudo foi verificar os efeitos do treinamento resistido na flexibilidade de idosas (grupo de força, GF, $\mathrm{n}=11, \mathrm{X}=66,3 \pm 7,84$ anos/grupo controle, $\mathbf{G C}, \mathrm{n}=11, \mathrm{X}=65,1 \pm 3,33$ anos). Elas foram submetidas a um treinamento resistido de força (75-85\% 1RM) por 16 semanas, 2 dias/semana. O tratamento estatístico utilizado foi o teste " $t$ " de Student pareado, através do programa SPSS 10.0 for Windows. Os testes mostraram resultados significativos do GF no ganho da força máxima e na flexibilidade. Os dados sugerem que o programa de treinamento pode melhorar o desempenho das atividades da vida diária da amostra com um treinamento de apenas 2 dias/semana.

Palavras-chave - treinamento resistido, força, flexibilidade, autonomia, idoso.

Endereço para correspondência:

Rua Oscar Clark, 805 - Pq. Mataruna - CEP 28970-000 - Araruama - RJ

3




\section{Effects of resistance training on flexibility of elderly women}

This study aimed to investigate the effects of resistance training on flexibility of the elderly women (strength group, SG, $\mathrm{n}=11, \mathrm{X}$ $=66.3 \pm 7.84$ years old/control group, CG, $n=11, X=65.1$ \pm 3.33 years old). They participated in a resistance training (75$85 \%$ 1RM) for 16 weeks, 2 days a week. Statistic treatment used was paired sample " $t$ " test, using a SPSS 10.0 for Windows program. Tests showed significant results of the $\mathbf{S G}$ in the maximum strength and flexibility. These data suggest that training programs can improve the performance of activities of daily life with a training of only 2 days a week.

Keywords - resistance training, strength, flexibility, autonomy, elderly.

\section{Efectos del entrenamiento de fuerza en la flexibilidad de mujeres mayores}

El objetivo de este estudio fue a verificar los efectos del entrenamiento resistido en la flexibilidad de mayores (grupo de fuerza, $G F, n=11, X=66,3 \pm 7,84$ años/grupo control, $G C, n=11$, $X=65,1 \pm 3,33$ años). Ellas habían sido sometidas a un entrenamiento resistido de fuerza (75-85\% 1RM) por 16 semanas, 2 días/semana. El tratamiento estadístico utilizado fue el test " $\mathrm{t}$ " de Student pareado, a través del programa SPSS 10.0 fuere Windows. Los tests mostraron resultados significativos del GF en la ganancia de la fuerza máxima y en la flexibilidad. Los datos sugieren que lo programa de entrenamiento puede mejorar el desempeño de las actividades de la vida diaria de la muestra con un entrenamiento de sólo 2 días/semana.

Palabras clave - entrenamiento resistido, fuerza, flexibilidad, autonomía, mayor.

\section{INTRODUÇÃO}

As modificações relacionadas com o envelhecimento sobre $\circ$ sistema musculoesquelético constituem, talvez, uma maior fonte de preocupação para os idosos (ROBERGS; ROBERTS, 2002).

Para Weineck (2000), a diminuição da massa muscular e da força muscular é uma das manifestações mais conhecidas nessa fase da vida. Esta perda, chamada de sarcopenia, se mostra como um importante fator de contribuição para a redução da capacidade funcional no envelhecimento. Devido a esse processo, os indivíduos vão gradualmente perdendo a força, o que dificulta a execução das atividades diárias (FRONTERA; BIGARD, 2002).

Posner et al. (1995) afirmam que o treinamento de força aumenta o desempenho das atividades da vida diária. De acordo com o ACSM (2003), o treinamento resistido de força ajuda a preservar e a aprimorar esta qualidade física nos indivíduos mais velhos. Isso pode prevenir quedas, melhorar a mobilidade e contrabalançar a fraqueza e fragilidade muscular.

Weineck (1999) aponta que o desenvolvimento da força e da flexibilidade é muito significativo na melhora da saúde e do bem-estar de qualquer sujeito. E Dantas et al. (2002) afirmam que a flexibilidade, conjugada com a força, permite ao idoso desincumbir-se das tarefas diárias com reduzido risco de lesões. Portanto, a manutenção da força muscular, ou o seu aprimoramento, permite a qualquer indivíduo executar as tarefas da vida diária com menos estresse fisiológico (ACSM, 2003).

Dantas (2002) reporta que uma motricidade equilibrada realça o corpo, valoriza o auto-conceito e a auto-estima, criando, ao mesmo tempo, uma interdependência produtiva e saudável com o meio ambiente, com amigos, familiares e a faixa social a que o idoso pertence. Novaes et al. (2002) corroboram com esses apontamentos, reforçando que a estética corporal não pode ser interpretada meramente como uma expressão de vaidade. A agregação desse valor para o idoso pode proporcionar a meIhora da auto-imagem e da auto-estima, e, por conseqüência, da qualidade de vida.

\section{OBJETIVO}

O objetivo deste estudo foi investigar os efeitos de um treinamento resistido na força máxima e na flexibilidade de um grupo de idosas, aparentemente saudáveis, participantes de um programa de treinamento na Academia Capacidade Vital, situada na cidade de Araruama, estado do Rio de Janeiro, e comparar com um grupo controle.

\section{METODOLOGIA}

\section{Amostra}

A população pesquisada se encontra na faixa etária de 60 a 88 anos de idade, do sexo feminino, com pessoas fisicamente independentes, segundo a classificação de Spirduso (1995). No primeiro momento, foram selecionadas, de forma intencional, 22 idosas para constituírem a amostra. No segundo momento, este grupo foi subdividido, randomicamente, em um grupo controle (GC, $n=11, X=65,1 \pm 3,33$ anos) e um grupo de treinamento resistido de força (GF, $n=11, X=66,3 \pm 7,84$ anos), para ser submetido ao tratamento experimental.

\section{Procedimentos}

O teste de carga máxima de 1 repetição (1RM) (BAECHLE; GROVES, 1992) foi usado para avaliar a força máxima, nos exercícios supino reto (SR), leg press (LP), puxada por trás (PT), hack $45^{\circ}$ (HACK), rosca bíceps com halteres (RB), extensão de 
pernas (EP), e rosca tríceps no pulley (RT). Foi aplicado o teste angular de avaliação da flexibilidade pelo protocolo LABIFIE de goniometria (DANTAS, 1999) nos seguintes movimentos: abdução de ombro (AO), flexão do quadril (FQ), extensão do quadril (EQ) e flexão de joelho (FJ).

Todos os testes foram realizados no início e no final do estudo, em ambos os grupos (GC e GF). O GF se submeteu, voluntariamente, a um programa de treinamento resistido de força de 75 a $85 \%$ de 1RM (WESTCOTT; BAECHLE, 2001), por um período de 16 semanas, 2 dias/semana. Antes da realização dos testes de 1RM, os sujeitos da pesquisa realizaram 4 sessões de treinamento para aprendizagem dos exercícios do programa (PLOUTZ-SNYDER; GIAMIS, 2001).

Os dados coletados foram analisados por meio de estatística descritiva e inferencial (teste " $\mathrm{t}$ " de Student), através do programa SPSS 10.0 for Windows.

O grupo selecionado assinou o termo de participação consentida, conforme resolução 196, de 10 de outubro de 1996, do Conselho Nacional de Saúde para as normas éticas de pesquisas envolvendo seres humanos.

\section{Instrumentos}

Como instrumentos de avaliação dos testes e da aplicação do tratamento experimental para este estudo, foram adotados: aparelho aglomerado de musculação (Apolo com placas, Brasil); pesos livres de halteres (Body and Sould, Brasil); cadeira extensora com placas (Apolo, Brasil); hack $45^{\circ}$ de anilhas (Physicus, Brasil) e um goniômetro de aço $360^{\circ}$ (Cardiomed, Brasil).

\section{Tratamento Experimental}

O GC manteve seus afazeres diários normais em todo o período do estudo, respeitando-se aqueles que costumavam fazer suas caminhadas matinais. Este grupo se comprometeu em não fazer nenhuma atividade física sistematizada que envolvesse trabalhos de força e/ou de flexibilidade durante as dezesseis semanas de experimento, até a realização do pós-teste.

O GF treinou os mesmos exercícios do teste de força máxima com aproximadamente $50 \%$ de 1 RM, com uma série do tipo alternada por segmento, nas primeiras 4 semanas. Após o período de adaptação, o GF treinou na fase específica utilizando $2 \times 8$ a 10 repetições, com intervalos de um a dois minutos entre as séries e entre os exercícios. A carga esteve entre 75 a $85 \%$ de 1 RM. A velocidade de execução foi de lenta a moderada. Os exercícios e o tipo de série foram mantidos.

O controle da carga de treinamento foi feito através do recurso proposto por Baechle; Groves (1992, apud NOVAES; VIANNA, 2003), denominado de "regra de dois por dois". Consiste no ajuste da carga toda vez que o indivíduo realizar um determinado exercício no limite superior (10 repetições) por duas sessões seguidas.

Tabela 01: Características da Amostra GF $(n=11)$ x GC $(n=11)$

\begin{tabular}{lccc}
\hline & Peso & Estatura & IMC \\
\hline GF & $63,36 \pm 10,60$ & $1,537 \pm 0,065$ & $26,70 \pm 3,545$ \\
GC & $64,59 \pm 7,16$ & $1,504 \pm 0,042$ & $28,52 \pm 2,486$ \\
Teste " $\mathrm{t}$ " ind. & 0,3183 & $-1,4119$ & $-1,3914$ \\
Valor de "p" & 0,7536 & 0,1734 & 0,1794 \\
\hline
\end{tabular}

$p<0,05 t_{\text {crítico }}=2,086$

Tabela 2: Médias dos Testes de 1RM GF x GC

\begin{tabular}{lccccccc}
\hline Testes & SR & LP & PT & HACK & RB & EP & RT \\
\hline GFpré & $16,9 \pm 4,7$ & $46,4 \pm 12,1$ & $27,8 \pm 9,1$ & $42,7 \pm 16,8$ & $5,9 \pm 1,1$ & $30,0 \pm 7,1$ & $11,7 \pm 2,5$ \\
GFpós & $22,5 \pm 5,7$ & $69,1 \pm 21,2$ & $36,1 \pm 7,4$ & $81,8 \pm 16,6$ & $8,2 \pm 1,1$ & $42,7 \pm 6,5$ & $16,4 \pm 2,5$ \\
"t" par. & 6,080 & 5,061 & 6,899 & 6,748 & 11,656 & 6,957 & 7,455 \\
GCpré & $16,0 \pm 4,4$ & $47,3 \pm 13,5$ & $25,3 \pm 2,8$ & $49,1 \pm 11,4$ & $4,4 \pm 0,5$ & $24,5 \pm 4,7$ & $12,3 \pm 2,1$ \\
GCpós & $15,8 \pm 4,9$ & $49,1 \pm 12,2$ & $26,5 \pm 4,7$ & $52,7 \pm 9,1$ & $4,7 \pm 0,5$ & $27,7 \pm 3,4$ & $12,8 \pm 2,4$ \\
"t" par. & 0,265 & 1,491 & 1,491 & 1,789 & 1,936 & 2,609 & 1,000 \\
\hline
\end{tabular}

$t_{\text {crítico }}=2,228$

$p<0,05$

Tabela 3: Médias dos Testes de Goniometria (graus) GF x GC

\begin{tabular}{lcccc}
\hline Testes & AO & FQ & EQ \\
\hline GF pré & $184,2^{\circ} \pm 7,49$ & $99,2^{\circ} \pm 11,94$ & $21,1^{\circ} \pm 6,55$ & FJ \\
GF pós & $190,5^{\circ} \pm 6,06$ & $112,4^{\circ} \pm 9,89$ & $30,8^{\circ} \pm 7,61$ & $141,5^{\circ} \pm 9,26$ \\
Teste "t" par. & 4,494 & 4,651 & 5,452 & $146,3^{\circ} \pm 7,06$ \\
GC pré & $179,6^{\circ} \pm 7,15$ & $102,9^{\circ} \pm 14,78$ & $19,3^{\circ} \pm 3,93$ & 2,962 \\
GC pós & $180,4^{\circ} \pm 6,01$ & $97,8^{\circ} \pm 13,15$ & $17,4^{\circ} \pm 5,32$ & $142,1^{\circ} \pm 4,35$ \\
\hline Teste " $t$ " par. & 0,798 & $-1,741$ & $-1,505$ & $141,5^{\circ} \pm 3,70$ \\
\hline
\end{tabular}

$t_{\text {crítico }}=2,228$

$p<0,05$ 
A respiração usada foi do tipo "passivo-eletiva" (WESTCOTT; BAECHLE, 2001) por ser a mais indicada para o grupo etário. Consiste na respiração contínua, expirando na fase concêntrica do movimento e inspirando na fase excêntrica.

Ambas as fases, de adaptação e específica, tiveram dez minutos de aquecimento, com exercícios que envolveram a mobilidade das principais articulações. $\bigcirc$ relaxamento foi de cinco minutos com exercícios de alongamento em níveis submáximos (DANTAS, 1999) ao término de cada sessão.

\section{RESULTADOS E DISCUSSÃO}

Os resultados da presente pesquisa serão apresentados e discutidos em três grupos distintos: características da amostra, comparação da força máxima GC x GF (pré e pós-teste) e comparação da flexibilidade GC x GF (pré e pós-teste).

\section{Características da Amostra}

Inicialmente, a amostra (GF e GC) apresentou as características antropométricas, que podem ser observadas na tabela 1 .

Analisando-se a tabela 1, pode-se observar que quanto às características antropométricas de estatura, peso e índice de massa corporal (IMC), os grupos de força (GF) e de controle (GC) apresentaram médias similares para o teste " $t$ " de amostras independentes, ou seja, não houve diferença significativa entre as médias.

Esses dados indicam que os grupos analisados eram homogêneos, pois $O$ " $t$ " encontrado foi menor que $O$ " $t$ " crítico para $p<0,05$. Apenas seria admitido a não homogeneidade dos grupos se fosse obtido um valor $\mathrm{p}<0,05$, o que não ocorreu.

\section{Comparação da Força Máxima GC x GF}

Os resultados do estudo quanto à força podem ser observados na tabela 2 .

Analisando-se a tabela 2, pode-se verificar que foi encontrada uma melhora significativa do grupo de treinamento resistido de força (GF) do pré para o pós-teste. Foram observadas diferenças significativas quando se comparou o GC com o GF.

Os resultados sugerem que o GF pode minimizar a perda da massa muscular com o envelhecimento e, com isso, obter melhoras na autonomia funcional e na qualidade de vida. $\bigcirc$ mesmo resultado não ocorreu com o GC. A exceção foi no exercício de extensão de pernas (EP), que obteve uma diferença significativa. Isso pode ser explicado pela prática das caminhadas rotineiras, que foi relatado por alguns elementos do GC.

Este estudo se propôs a realizar o treinamento de força com uma freqüência de dois dias por semana, baseado nos apontamentos de Craig (2002), que relatam que as mulheres após os 50 anos de idade, no período da menopausa, começam a apresentar declínios funcionais.
Dentre eles está a capacidade de regeneração do musculoesquelético (MATSUDO, 2001), que também é afetada pelo enveIhecimento. De acordo com Cannon (1998, apud MATSUDO, 2001), entre os fatores que influenciam essa recuperação estão o fator de crescimento do fibroblasto, o fator de crescimento insulinóide (IGF) e o fator de crescimento nervoso. Esses são importantes reguladores das células de crescimento e diferenciação precursoras do músculo, e manutenção ou estabelecimento do contato neural.

Por essa razão, então, foi determinado um intervalo mínimo entre as sessões de 72 horas, embora a maioria dos estudos reporte uma freqüência semanal de três vezes por semana, para um treinamento de força. Porém, Evans (1999) e Porter et al. (2002) demonstraram resultados positivos com apenas 2 dias/semana. No estudo de Stadler et al. (1996), foram observados que os incrementos de força muscular obtidos em mulheres idosas foram similares quanto à freqüência semanal. Pois, um grupo realizou um treinamento de força com freqüência de três vezes por semana, e o outro treinou com duas vezes por semana. Ambos atingiram aproximadamente um incremento de força de 28,5\% e $27 \%$ respectivamente. Os resultados comparativos mostraram que a diferença não foi significativa.

Esses dados podem explicar os resultados positivos aqui encontrados com um treinamento de força de apenas 2 dias/semana, pois os sujeitos participantes do GF obtiveram um aumento dos níveis da força máxima entre 30 e 75\% de 1 RM. Esse incremento na força máxima também foi encontrado nos estudos de Porter et al. (2002). Outras pesquisas mostram que incrementos de força com programas de alta intensidade podem chegar a melhoras de até $227 \%$ de 1 RM (EVANS, 1996, 1999; RASO et al., 1997). Entretanto, num programa de baixa intensidade, os ganhos giram em torno de $20 \%$ num mesmo período de treinamento (MATSUDO, 2001).

Os achados desta pesquisa estão de acordo com estudos de Fiatarone-Singh (1998, apud MATSUDO, 2001), que indicam que um treinamento de força de alta intensidade na terceira idade é seguro e muito mais efetivo que o treinamento de baixa intensidade, para que ocorram as adaptações musculares.

As recomendações de percentual de carga a ser trabalhado em treinamentos de força estão na faixa de 60 a $85 \%$ de 1 RM (WILLOUGHBY, 2003). Esse percentual de intensidade foi usado em outras pesquisas (CARPINELLI; OTTO, 1998; GERALDES, 2000; KRAEMER et al., 1997; PORTER et al., 2002; SCHOT et al., 2003; STADLER et al., 1996; VALE, 2004). Em todas elas, os ganhos da força máxima foram significativos. Esses dados confirmam os resultados positivos que foram encontrados neste estudo, pois a intensidade da carga utilizada foi de 75 a $85 \%$ de IRM, portanto, dentro dessa faixa percentual.

Na fase específica do treinamento resistido de força, o GF realizou o programa de exercícios com duas séries $(2 \times 8$ a 10 repetições de 1RM). Essa prescrição vai de encontro às recomendações de Feigenbaum; Pollock (1999) e Willoughby (2003). Os resultados positivos aqui achados corroboram com estudos de Carpinelii; 
Otto (1998), que não encontraram diferenças significativas entre programas de duas para três séries $(2 \times 10$ para $3 \times 10$ repetições de 1RM).

O estudo de Geraldes (2000) apontou ganhos significativos de força máxima de até $36 \%$, aproximadamente. O grupo de idosas realizou um treinamento contra resistência com a utilização de um programa de três séries $(3 \times 8$ a 10 repetições de $1 R M)$, três vezes por semana, durante doze semanas. Enquanto que, nesta pesquisa, os resultados foram superiores, apresentando ganhos na força máxima de até $75 \%$ aproximadamente, e com treinos de $2 \times 8$ a 10 repetições de 1RM, 2 dias/semana.

Embora neste estudo não tenha havido recursos para pesquisas com métodos invasivos ou mais sofisticados, o aumento da massa muscular e da força muscular tem se mostrado maior nas fibras do tipo Illb, proporcionando hipertrofia e ganhos de força (HORTOBAGYl et al., 2002). Nessa faixa etária, esses incrementos são muito necessários para a autonomia funcional do idoso (DANTAS, 1998; LAREW et al., 2003; MATSUDO, 2001; McARDLE et al., 1998; NEWTON et al., 2002).

O treinamento resistido de força pode trazer inúmeros benefícios aos gerontes. Dentre eles, se destacam a manutenção da musculatura e do metabolismo, ganho do tecido muscular, redução da gordura corporal, aumento da densidade mineral óssea, melhoria do metabolismo de glicose e dos lipídeos sangüíneos, redução da pressão arterial, melhora da postura, mobilidade e flexibilidade (BRANDON et al., 2004; DE VITO et al., 2003; REEVES et al., 2004; TAYLOR, 2003; WESTCOTT; BAECHLE, 2001).

\section{Comparação da Flexibilidade GC x GF (pré e pós-teste)}

Os resultados quanto à flexibilidade podem ser observados na tabela 3.

Analisando-se a tabela 3, pode-se verificar que o treinamento proporcionou melhora significativa dos níveis de flexibilidade no GF do pré para o pós-teste.

Quando comparado o aumento dos níveis de flexibilidade do grupo de força (GF) com o grupo controle (GC), notou-se que o GF obteve um aumento expressivo das médias dos ângulos dos movimentos analisados. $O G C$ não teve alteração significativa. Os resultados sugerem que o GF pôde obter melhoras na autonomia funcional e na qualidade de vida.

No estudo de Wood et al. (2001) foram encontrados resultados positivos nos níveis de flexibilidade do grupo que treinou força com $75 \%$ de 5 RM, com $2 \times 8$ a 12 repetições. A similaridade desse estudo com a presente pesquisa esteve nos resultados alcançados, no número de séries e na progressão de cargas, visto que a carga sofria um aumento de cerca de $10 \%$ quando os sujeitos conseguiam realizar as duas séries no limite superior das repetições.

Os resultados do corrente estudo são corroborados pela pesquisa de Adams et al. (2001), que apresentou índices positivos medi- dos no teste de sentar e alcançar. É importante ressaltar que a freqüência semanal também foi de apenas 2 dias/semana, e que o grupo de força treinou com 2 a 3 séries de 8 a 10 repetições, com 70 a $80 \%$ de 1 RM, por oito semanas.

Os achados positivos no ganho dos níveis de flexibilidade do grupo de força dessa pesquisa, realizada com um treinamento resistido de força, se assemelham com as recomendações e pesquisas de treinamentos específicos de flexibilidade (ACHOUR JÚNIOR, 1999; CONCEIÇÃO, 2004; DANTAS, 1999; KLEIN, 2003; SCHROEDER, 2003; THACKER et al., 2004; VALE et al., 2003a; VALE, 2004; VAREJÃO et al., 2004; WIEMANN; KLEE, 2000).

A redução da flexibilidade, acompanhada do envelhecimento, pode provocar comprometimento com perda parcial da independência dos movimentos já no início da idade avançada. Isso pode trazer prejuízos a autonomia funcional do idoso (ACHOUR JÚNIOR, 1999; SCHROEDER, 2003; VALE et al., 2003b).

\section{CONCLUSÃO}

O treinamento resistido de força proporcionou mudanças positivas no aspecto físico/funcional e psicológico/auto-estima para $a$ amostra. Para $p<0,05$, a força e a flexibilidade foram aumentadas significativamente em todos os exercícios e amplitudes de movimento.

O tema abordado merece mais investigações para esclarecer as possíveis dúvidas geradas por esta pesquisa. Porém, pelo aumento dos níveis de força máxima e de flexibilidade alcançados pelo GF, este estudo sugere que o treinamento resistido de força, com freqüência de 2 dias/semana, de forma sistematizada, pode tornar possível a realização das atividades da vida diária com menos esforço e prolongar a independência funcional. Assim, nos últimos anos de vida, o idoso pode ter a possibilidade de viver de uma maneira auto-suficiente e dignificada.

\section{REFERÊNCIAS}

ACHOUR JÚNIOR, A. Bases para exercícios de alongamento relacionado com a saúde e o desempenho atlético. $2^{a}$ edição. Londrina: Phorte editora, 1999.

ADAMS, K. J.; SWANK, A. M.; BERNING, J. M.; SEVENE-ADAMS, P. G.; BARNARD, K. L.; SHIMP-BOWERMAN, J. Progressive strength training in sedentary, older African American women. Medicine and Science in Sports and Exercise. v. 33, n. 9, p. 1567-1576, 2001.

AMERICAN COLLEGE OF SPORTS MEDICINE. Diretrizes do ACSM para os testes de esforço e sua prescrição. $6^{a}$ edição. Rio de Janeiro: Guanabara Koogan, 2003.

ARAGÃO, Jani C. B. Efeitos da resistência muscular localizada visando a autonomia e a qualidade de vida de idosos. 2002, 332 f. Dissertação (Mestrado em Ciência da Motricidade Humana). Universidade Castelo Branco - UCB. RJ.

BAECHLE, T. R.; GROVES, B. R. Weight training: steps to success. Champaign: Human Kinetics, 1992.

BRANDON, L. J.; BOYETTE, L. W.; LLOYD, A.; GAASCH, D. A. Resistive training and longterm functional in older adults. Journal of Aging and Physical Activity, v. 12, p. 10-28, 2004.

BRASIL. Ministério da Saúde. Resolução 196/96. O Plenário do Conselho Nacional de Saúde resolve aprovar diretrizes e normas regulamentadoras de pesquisas envolvendo seres humanos. Em 10 de outubro de 1996.

CARPINELLI, R. N.; OTTO, R. M. Strength training: single versus multiple sets. Sports and Medicine, 26(2), p. 73-84, Aug 1998. 
CONCEIÇÃO, Mário Cezar de Souza Costa. Efeitos de quatro diferentes tempos de insistência de flexionamento estático na flexibilidade de adultos jovens. 2004, 117 f. Dissertação (Mestrado em Ciência da Motricidade Humana). Universidade Castelo Branco - UCB. Rio de Janeiro.

CRAIG, B. W. Resistance training and bone growth in the elderly. National Strength and Conditioning Association, v. 24, n 3, p. 63-64, June 2002.

DANTAS, Estélio H. M. A Prática da Preparação Física. $4^{a}$ ed. Rio de Janeiro: Shape 1998 1999

Flexibilidade: alongamento e flexionamento. $4^{a}$ edição. Rio de Janeiro: Shape,

Fitness, saúde, wellness e qualidade de vida. Revista Mineira de Educação Física v. 10, $\mathrm{n}^{\circ} 1$, p. 106-150, 2002.

DANTAS, E. H. M.; PEREIRA, S. A. M.; ARAGÃO, J. C. B.; OTA, A.. H. Perda da flexibilidade no idoso. Fitness e Performance Journal. v. 1, $n^{\circ} 3$, p. 12-20, 2002.

DE VITO, C. A.; MORGAN, R. O.; DUQUE, M.; ABDEL-MOTY, E.; VIRNIG, B. A. Physica performance effects of low-intensity exercise among clinically defined high-risk elders. Gerontology, 49, 3; p. 146-154, May/Jun, 2003

EVANS, William J. Reversing sarcopenia: how weight training can build strength and vitality. Geriatrics, v. 81, $n^{\circ} 5$, p. 46-53,1996.

Exercise training guidelines for the elderly. Medicine and Science in Sports and Exercise, v. 31, $n^{\circ} 1$, p. 12-17, 1999.

FEIGENBAUM, M. S.; POLLOCK, M. L. Prescription of resistance training for health and disease. Medicine and Science in Sports and Exercise. v. 31, n. 1, p. 38-45, 1999

FRONTERA, W. R.; BIGARD, X. The benefits of strength training in the elderly. Science and Sports, v. 17, i 3, p. 109-116, May 2002.

GERALDES, A. A. R. Efeitos do treinamento contra resistência sobre a força muscular e o desempenho de habilidades funcionais selecionadas em mulheres. 2000, 233f. Dissertação (Mestrado em Ciência da Motricidade Humana), Universidade Castelo Branco, UCB, RJ.

HORTOBÁGYI, T.; MONEY, J.; ZHENG, D.; DUDEK, R.; FRASER, D.; DOHIN, L. Muscle adaptations to 7 days of exercise in young and older humans: eccentric overload versus standard resistive training. Journal of Aging and Physical Activity, 10, p. 290-305, 2002.

KLEIN, D. A. Flexibility in aging: stretching to mend the bend. American College of Sports Medicine Fit Society. p. 5,11, Summer 2003.

KRAEMER, J. B.; STONE, M. H.; O `BRYANT, H. S.; CONLEY, M. S.; JOHNSON, R. L.; NIEMAN, D. C.; HONEYCUTT, D. R.; HOKE, T. P. Effects of single vs. multiple sets of weight training: impact of volume, intensity, and variation. Journal Strength and Conditioning Research, 11(3), p. 143-147, 1997.

LAREW, K.; HUNTER, G. R.; LARSON-MEYER, D. E.; NEWCOMER, B. R.; MCCARTHY, J. P.; WEINSIER, R. L. Muscle metabolic function, exercise performance, and weight gain. Medicine and Science in Sports and Exercise, v. 35, n 2, p. 230-236, 2003.

MCARDLE, W. D.; KATCH, F. I.; KATCH, V. L. Fisiologia do exercício: energia, nutrição e desempenho físico. $4^{\text {a }}$ Ed. Rio de Janeiro: Guanabara Koogan, 1998.

MATSUDO, S. M. Envelhecimento e atividade física. Londrina: Midiograf, 2001

MUNN, J.; BEARD, D. J.; REFSHAUGE, K. M.; LEE, R. Y. W. Eccentric muscle strength in functional ankle instability. Medicine and Science in Sports and Exercise, v. 35, $n^{\circ} 2, p$ $245-250,2003$

NEWTON, R. U.; HÄKKINEN, K.; HÄKKINEN, A.; MCCORMICK, M.; VOLEK J.; KRAEMER, W.J. Mixed-methods resistance training increases power and strength of young and older men. Medicine and Science in Sports and Exercise, v. 34, n. 8, p. 1367-1375, 2002.

NOVAES, Jefferson S.; NOVAES, Sonia R. S.; MARQUES, Martim Bottaro; OLIVEIRA, Ricardo J. Vídeos de ginástica: a cultura dos corpos nas academias: uma análise fílmica. Fitness e Performance Journal, v. 1, n 5, p. 12-16, set/out 2002

NOVAES, Jefferson S.; VIANNA, Jefferson M. Personal training e condicionamento físico em academia. Rio de Janeiro: Shape, 2003.

PLOUTZ-SNYDER, L. L.; GIAMIS, E. L. Orientation and familiarization to 1RM strength testing in old and young women. Journal of Strength and Conditioning Research, 15(4), p. $519-523,2001$
PORTER, M. M.; NELSON, M. E.; FIATARONE SINGH, M. A.; LAYNE, J. E.; MORGANTI, C. M.; TRICE, I.; ECONOMOS, C. D.; ROUBENOFF, R.; EVANS, W.J. Effects of long-term resistance training and detraining on strength and physical activity in older women. Journal of Aging and Physical Activity, 10, p. 260-270, 2002.

POSNER, J. D.; MCCULLY, K. K.; LANDSBERG, L. A.; SANDS, L. P.; TYCENSKI, P.; HOLFMANN, M. T.; WETTERHOLT, K. L.; SHAW, C. E. Physical determinants of independence in mature women. Archive of Physical Medicine and Rehabilitation. v. 76, p. 373-380, 1995.

RASO, V.; ANDRADE, E. L. A.; MATSUDO, S. M. M.; MATSUDO, V. K. R. Exercícios com pesos para mulheres idosas. Revista Brasileira de Atividade Física e Saúde, v. 2, $\mathrm{n}^{\circ} 4$, p. 17-26, 1997

REEVES, N. D.; NARICI, M. V.; MAGANARIS, C. N. Effect of resistance training on skeletal muscle-specific force in elderly humans. Journal of Applied Physiology, v. 96, p. 885892,2004

ROBERGS, R. A.; ROBERTS, S. O. Princípios fundamentais de fisiologia do exercício para a aptidão, desempenho e saúde. $1^{a}$ edição. São Paulo: Phorte editora, 2002.

SCHOT, P. K.; KNUTZEN, K. M.; POOLE, S. M.; MROTEK, L. A. Sit-to-stand performance of older adults following strength training. Research Quarterly for Exercise and Sport, v. 74, $n^{\circ} 1$, p. 1-8, Mar, 2003.

SCHROEDER, J. Older adults: look at lifestyle to increase activity. American College of Sports Medicine Fit Society Page. p. 3-4, Summer 2003.

SPIRDUSO, Waneen, W. Physical dimensions of aging. Champaign: Human Kinetics, 1995.

STADLER, L. V.; STUBBS, N. B.; VUKOVICH, M. D. A comparison of a 2-day and 3-day per week resistance training program on strength gains in older adults (Abstract). Medicine and Science in Sports and Exercise, supplement, v. 29, $n^{\circ}$ 5, p.S254, 1996.

TAYLOR, A. C.; MCCARTNEY, C. N.; KAMATH, M. V.; WILEY, R. L. Isometric training lowers resting blood pressure and modulates autonomic control. Medicine and Science in Sports and Exercise, v. 35, $\mathrm{n}^{\circ} 2$, p. 251-256, 2003

THACKER, S. B.; GILCHRIST, J.; STROUP, D. F.; KIMSEY, C. D., JR. The impact of stretching on sports injury risk: a systematic review of the literature. Medicine and Science in Sports and Exercise, v. 36, n. 3, p. 371-378, 2004.

VALE, R. G. S. Efeitos do treinamento de força e de flexibilidade sobre a autonomia e qualidade de vida de mulheres senescentes. 2004, 219 f. Dissertação (Mestrado em Ciência da Motricidade Humana). Universidade Castelo Branco - UCB. Rio de Janeiro.

VALE, R. G. S.; ARAGÃO, J. C. B.; DANTAS, E. H. M. A flexibilidade na autonomia funcional de idosas independentes. Fitness e Performance Journal, v. 2, $n^{\circ} 1$, p. 23-29, 2003a

VALE, R. G. S.; VAREJÃO, R. V.; DANTAS, E. H. M. A flexibilidade na senescência. In: DANTAS, E. H. M.; OLIVEIRA, R. J. (Org.). Exercício, maturidade e qualidade de vida. $2^{a}$ ed. Rio de Janeiro: Shape, 2003b.

VAREJÃO, Ronaldo V.; MELO, Roberto; BARROS, Rosilane; VALE, Rodrigo G. S.; ARAGÃO, Jani C. B.; AMORIM, Fátima S.; DANTAS, Estélio H. M. Comparação dos efeitos do alongamento e do flexionamento ambos passivos sobre os níveis de flexibilidade, autonomia e qualidade de vida do idoso. FIEP Bulletin, v. 74, 2004.

WEINECK, J. Treinamento ideal. $9^{a}$ Ed. São Paulo: Manole, 1999.

Biologia do esporte. $2^{a}$ Ed. São Paulo: Manole, 2000.

WESTCOTT, W.; BAECHLE, T. Treinamento de força para a terceira idade. $1^{a}$ ed., São Paulo: Manole Editora, 2001

WIEMANN, K.; KLEE, A. Stretching e prestazione sportive di alto livello. Rivista di Cultura Sportiva SDS. a. XIX. $n^{\circ} 49$, p. 9-15, Aug-Set. 2000

WILLOUGHBY, D. S. Resistance training in older adult. American College of Sports Medicine Fit Society Page. p. 8-9, Summer 2003

WOOD, R. H.; REYES, R.; WELSCH, M. A.; FAVALORO SABATIER, J.; SABATIER, M.; LEE, C. M.; JOHNSON, L. G.; HOOPER, P. F. Concurrent cardiovascular and resistance training in health older adults. Medicine and Science in Sports and Exercise (Hagerstown, Md.) $33(10)$, p. $1751-1758$, Oct 2001 